\title{
Crítica a los mitos acerca de las migraciones centroamericanas a la frontera sur de México
}

\author{
Rodolfo Casillas R. \\ Manuel Ángel Castillo G. \\ Laura Muñoz R. \\ Centro de Estudios Demográficos y de Desarrollo Urbano \\ El Colegio de México
}

\begin{abstract}
Resumen
Este trabajo intenta plantear y enfocar los distintos fenómenos migratorios que ocurren en la frontera sur de México. Como punto de partida, presenta algunas de las ideas que han proliferado en torno a las migraciones hacia la frontera sur de México, muchas de las cuales son comunes a las de otros contextos fronterizos. Como parte de un proyecto de investigación desarrollado se hace uso de las indagaciones de campo realizadas en la región del Soconusco, en el Estado de Chiapas. En base a los resultados debe enfatizarse que la carencia de conocimientos requiere de estudios más profundos y diversos enfocados al análisis de otros flujos migratorios y se puede concluir que la forma adecuada de enfrentar este fenómeno no radica en las medidas de control, sino más bien en la oportuna contribución para solucionar los problemas de origen.
\end{abstract}

Palabras clave: migración centroamericana, frontera sur de México, flujo migratorio, Soconusco, refugiado político.

\begin{abstract}
This project is focused on several migratory phenomena that continuously appear in the South border of Mexico. It starts by presenting some current points of view that have appeared around the migration in the Southern border of Mexico, same that show similar characteristics to other border contexts. As part of a research project that is already developed, it uses field researches performed in the region of Soconusco in Chiapas. Based on these output, it must be emphasized that the lack of knowledge requires deeper and more diverse researches with focus into the analysis of other migratory flow. It gets to the conclusion that the control measures are not the best nor the most proper ways to face this phenomenon, but on the accurate and timely contribution to solve the origin problems.
\end{abstract}

Keywords: Central America migration, South border of Mexico, migratory flow, Soconusco, political refugee. 


\title{
CRITICA A LOS MITOS ACERCA DE LAS MIGRACIONES CENTROAMERICANAS A LA FRONTERA SUR DE MEXICO
}

\author{
Rodolfo Casillas R. \\ Manuel Angel Castillo G. \\ Laura Muñoz R*
}

En trabajos anteriores (Casillas y Castillo, 1987) hemos señalado que la frontera sur de México ha sido objeto de atención reciente por parte de diferentes sectores (oficiales, privados, académicos ${ }^{1}$ ). Uno de los problemas que esta revalorización de la región enfrenta, es la carencia de estudios sistemáticos acerca de los distintos fenómenos que en ella ocurren; es decir, que a la complejidad y heterogeneidad de los mismos debemos sumar nuestra carencia de conocimientos sistemáticos.

Lo que se intenta plantear en este trabajo es que esta deficiencia tiene implicaciones más allá del ámbito de las preocupaciones de índole académica. La falta de conocimiento preciso es caldo de cultivo para la difusión de creencias erróneas o, lo que es peor, de ideas que responden a intereses inconfesados. De esa cuenta, los mitos se extienden y circulan con una rapidez inaudita; pero lo que es más preocupante es que no solamente sorprenden y convencen a la población sino que, además, la comprometen en acciones de alcances insospechados, pues pueden dar lugar a la adopción de políticas y medidas oficiales.

Veamos, pues, algunas de las ideas que han proliferado en torno a las migraciones hacia la frontera sur de México. Muchas de ellas son

* Los dos primeros son investigadores y la segunda colaboradora del proyecto de investigación "Impactos regionales de las migraciones internacionales a la frontera sur de México. El caso de los trabajađores estacionales", que se realizó en el Centro de Estudios Demográficos y de Desarrollo Urbano de El Colegio de México con el auspicio del Consejo Nacional de Ciencia y Tecnología. El proyecto contó con la participación de un grupo de personas que colaboraron en diversas fases del estudio; sin embargo, es necesario dejar constancia expresa de las valiosas contribuciones de Rosa María Camarena y Emma Calderón, cuyos aportes forman parte de este trabajo.

${ }^{1}$ En rigor de justicia es necesario señalar el meritorio esfuerzo de varias instituciones en esta dirección; entre ellas, el Centro de Investigaciones Ecológicas del Sureste, la Universidad Autónoma de Chiapas, y el Centro de Investigaciones y Estudios Superiores de Antropología Social. 
comunes a las que se han extendido en otros contextos fronterizos y, sin ir más lejos, encontramos coincidencias con los conceptos manejados en los Estados Unidos respecto a las migraciones de origen hispano y, en particular, de procedencia mexicana. Para nuestra argumentación nos basaremos, en la medida de lo posible, en nuestras indagaciones realizadas en el terreno como parte de un proyecto de investigación desarrollado en la región del Soconusco en el Estado de Chiapas ${ }^{2}$.

I. La migración centroamericana a la frontera sur de México es de gran magnitud y prácticamente inunda el campo y las ciudades chiapanecas.

II.La llegada ininterrumpida de guatemaltecos (y de centroamericanos en general) incide negativamente en los índices de violencia, en el aumento de la prostitución, en la demanda creciente de servicios públicos, en la dotación de tierras y en el desplazamiento de la mano de obra local y nacional; todo lo cual redunda en una creciente inestabilidad social.

Estas tesis se basan en algunos supuestos no explícitos, que conviene al análisis llamar por su nombre:

1. "No hay que aceptar al desplazado; hay que cortar de tajo el flujo de migrantes y, sobre todo, no darles ocupación, para que, si los empleos son pocos, les toquen a los mexicanos".

2. "Antes de 1980 (época en que se inició el auge de la migración centroamericana) no había problemas sociales en la región

2 Se trata de recorridos de campo, las entrevistas, las observaciones y los resultados de una encuesta levantada a trabajadores migratorios, como parte del proyecto de investigación denominado "Impactos regionales de las migraciones internacionales a la frontera sur de México. El caso de los trabajadores estacionales", realizado en el Centro de Estudios Demográficos y de Desarrollo Urbano de El Colegio de México, bajo la responsabilidad de Rodolfo Casillas y Manuel Angel Castillo, y que contó con el patrocinio del Consejo Nacional de Ciencia y Tecnología (CONACYT). Por esa razón, en este trabajo nos circunscribimos a la dinámica fronteriza del estado de Chiapas y dejamos para trabajos posteriores las consideraciones referentes al resto de la región fronteriza, partiendo del supuesto de que su actividad e importancia en este sentido es bastante menor. 
fronteriza 0 , si los había, eran de alcances menores y no atentaban contra el orden social vigente".

3. "Todos los centroamericanos son iguales, es decir, gérmenes móviles de inestabilidad social (léase agitación odelincuencia); si no, icómo se explican sus relaciones con los problemas sociales?".

4. "Dichos migrantes no vienen a México ni pasan por él en calidad de desplazados de guerra, sino que son migrantes económicos o difusores de la revolución importada, esa que tanta pobreza y muerte ha traído a las poblaciones centroamericanas".

5. "Los mexicanos que apoyan a dichos migrantes son los interesados en fomentar la inestabilidad en el país a fin de sacar partido para sus intereses antinacionales".

6. "Existe una medición bastante precisa de los distintos flujos migratorios que permiten aseverar que su presencia en el área rural y en los núcleos urbanos son una causa fundamental del acelerado incremento demográfico observado en los años recientes".

\section{EL CASO DE LOS REFUGIADOS GUATEMALTECOS}

Uno de los flujos migratorios más importantes que han llegado en época reciente a la frontera sur de México, es el de los refugiados guatemaltecos ${ }^{3}$, de quienes se podría pensar que constituyen una de las razones que dieron lugar a los supuestos arriba mencionados, por ser demandantes de condiciones básicas de subsistencia. Lo cierto es que su llegada masiva (pues en muchos casos se trató de familias enteras, o lo que quedaba de ellas, y hasta incluso de comunidades completas) dio la pauta para la difusión de preocupaciones y alarmas, presentes en los mitos y en los supuestos entrecomillados anteriores.

La forma hasta cierto punto dramática en que se produjo el fenómeno de la llegada de los refugiados a territorio mexicano, llamó

${ }^{3}$ Las características de este flujo han sido documentadas no sólo por investigadores académicos (ver, entre otros, los trabajos de S. Aguayo, de los cuales es pertinente $E l$ éxodo centroamericano, CONAFE/SEP, 1a. ed., México, 1985), sino también por organismosy miembros de la comunidad internacional, especialmente relacionados con los programas de ayuda a refugiados. 
la atención de la comunidad internacional, que ha prestado su ayuda en la búsqueda de mejores condiciones de vida para personas que tuvieron que padecer hambre, enfermedades y persecución. En el ámbito local, diversos sectores también se incorporaron a esta tarea humanitariaygeneraron una corriente de opinión, no sin antes tener que vencer la campaña desinformadora de sus perseguidores y de algunos grupos locales que, directa o indirectamente, coincidieron con sus intereses ${ }^{4}$.

Uno de los resultados de la labor de apoyo a este grupo de desplazados ha sido la verificación de las condiciones que dieron lugar a este involuntario movimiento. Por supuesto que no se trata de personas que salieron de sus lugares de residencia habitual en busca de mejores condiciones de trabajoy de vida; se trata de una movilización forzada por circunstancias ajenas a su voluntad. Se ha comprobado que en el auge de la lucha contrainsurgente fueron muchos los sectores de población campesina que abandonaron sus reducidas propiedades; la prueba es que se encontraron, tiempo después, varios grupos deambulando por las montañas guatemaltecas en condiciones lamentables.

Los refugiados que pudieron llegar a México fueron los que corrieron con mejor suerte, puesto que recibieron atención médica, alimenticia y, en etapas posteriores, trabajo para la búsqueda de su autosuficiencia. En este punto creemos que es importante recalcar que, en general, todo migrante enfrenta la necesidad de resolver su situación de sobrevivencia en los lugares de llegada y eso se aplica no solamente al caso de los refugiados, comoveremos más adelante. Por eso pensamos que son equivocadas o insuficientes las tradicionales categorías polares de "migrantes económicos" y "perseguidos políticos" para clasificar los diversos tipos de migración que encontramos en el complejo contexto actuals.

El hostigamiento de que fueron objeto los refugiados localizados en algunos asentamientos del estado de Chiapas, sumado a presiones internas, dieron lugar a que el gobiernomexicano decidiera instrumentar un proceso de reubicación. Las medidas adoptadas tropezaron en

4 En este sentido es encomiable la labor de algunos grupos religiosos y, en particular, el papel desempeñado por la Diócesis de San Cristóbal de las Casas, Chiapas.

5 Sin embargo, este aspecto es motivo de una discusión amplia y específica que trasciende los alcances de este trabajo. 
un primer momento con algunas dificultades, pero concluyeron en la relocalización de aproximadamente el cuarenta por ciento de los refugiados (18,245 cifra de enero de 1986 según Aguayo y Dougherty, 1986: 271) hasta entonces atendidos hacia nuevos campamentos ubicados en los estados de Campeche y Quintana Roo ${ }^{6}$.

Por otra parte, nuestras observaciones de terreno nos permiten señalar que el impacto territorial del fenómeno de los refugiados es claramente perceptible. En un primer momento, el área de su asentamiento fue la parte norte del estado de Chiapas (ver mapas), a partir de la región Sierra; en ella se ubicaron los campamentos que fueron atendidos por las autoridades mexicanas y la comunidad internacional. No obstante, se dice que muchos más llegaron a la zona selvática, pero que las condiciones del terreno impedían su localización y atención adecuadas. Por otra parte, nuestra impresión es que la zona del Soconusco no constituyó lugar importante de llegada de este tipo de flujos; para ello nos basamos en el hecho de que los sistemas urbano y de comunicaciones, así como la estructura de tenencia y las formas de aprovechamiento de la tierraagrícola de la zona, fueron obstáculos para la integración permanente de personas con sus características.

\section{LAS MIGRACIONES A LOS CENTROS URBANOS}

Si observamos con cuidado la tesis y los supuestos mencionados al principio de este trabajo, se puede observar que en su mayoría, parten de -y se apoyan más bien en- una realidad urbana y de un posible impacto, hasta ahora no sustentado, de los migrantes que de manera temporal, de paso o definitiva, son vistos en centros urbanos o semiurbanos de Tapachula ${ }^{7}$ y en cabeceras municipales aledañas. La

${ }^{6} \mathrm{~A}$ la fecha se estima que el retorno de refugiados no excede de un total de 3,000 personas, dado que, según lo manifiestan en los documentos emitidos por sus propias organizaciones, el gobiernoguatemalteco no ha cumplido con las condiciones mínimas de seguridad y recepción para que se pueda dar un proceso de repatriación en el que se garantice su seguridad y el respeto a sus escasas pertenencias. El total se refiere al total máximo de refugiados localizados y registrados por la Comisión Mexicana de Ayuda a Refugiados (COMAR) y el Alto Comisionado de Naciones Unidas para la Ayuda de Refugiados (ACNUR).

${ }^{7}$ La ciudad de Tapachula es la segunda ciudad en tamaño e importancia del estado de Chiapas; es el "centro urbano" de la región del Soconusco, una de las más dinámicas y productivas de la entidad; además, cumple la función de centro fronterizo más importante, puesto que a ella confluye todo el tránsito de personas y el movimiento comercial de carácter internacional que se realiza a través de la entidad. 
llegada de estos flujos migratorios a territorio mexicano puede haber ocasionado un incremento en la demanda de servicios públicos, cosa común en el ámbito urbano, y de dotación de tierras, cuestión común en el campo, aunque este último aspecto lo veremos más adelante.

La idea general que se desprende de los registros oficiales de migración respecto a las migraciones a centros urbanos, es que es mayor el número de entradas al país por los puestos fronterizos del sur de México que el de salidas por los mismos (ver, por ejemplo, los datos del cuadro 1).

CUADRO 1. Distribución absoluta y relativa en las entradas y salidas de visitantes locales registradas en los puestos fronterizos del estado de Chiapas, según mes de ocurrencia, 1984.

\begin{tabular}{ccrcr}
\hline MES & ENTRADAS & $\%$ & SALIDA & \multicolumn{1}{c}{$\%$} \\
\hline Enero & 11,161 & 6.9 & 10,687 & 7.2 \\
Febrero & 12,479 & 7.7 & 11,318 & 7.6 \\
Marzo & 16,682 & 10.3 & 15,093 & 10.2 \\
Abril & 10,443 & 6.5 & 10,373 & 7.0 \\
Mayo & 10,734 & 6.6 & 10,787 & 7.3 \\
Junio & 8,368 & 5.2 & 7,416 & 5.0 \\
Julio & 12,784 & 7.9 & 12,012 & 8.1 \\
Agosto & 11,719 & 7.2 & 10,955 & 7.4 \\
Septiembre & 15,448 & 9.6 & 13,433 & 9.1 \\
Octubre & 17,250 & 10.6 & 14,762 & 10.0 \\
Noviembre & 18,692 & 11.6 & 16,566 & 11.1 \\
Diciembre & 15,982 & 9.9 & 14,934 & 10.0 \\
\hline T O T A L & 161,742 & 100.0 & 148,336 & 100.0 \\
\hline
\end{tabular}

Diferencias absolutas (entradas - salidas): 13,406

Fuente: Dirección General de Servicios Migratorios, Secretaría de Gobernación. Tabulaciones inéditas. México, 1985.

Aunque se reconoce que los registros no cubren el total de los movimientos migratorios internacionales, se puede decir que un 
porcentaje de los ingresados no retornan a Centroamérica y que además, en algunos casos, se modifica su estatuto de estadía, pues habiendo entrado documentados, al quedarse o ir a otro lugar, presumiblemente a Estados Unidos, lo hacen de manera indocumentada.

Por otra parte, se supone que es mayor el número de casos con estatuto de indocumentados desde el inicio de su migración, que el de los que se registran bajo una de las modalidades legalmente posibles por alguna autoridad migratoria, y que luego cambian su estatuto. Sin embargo, esto es más una apreciación que una comprobación empírica; no descalificamos la apreciación, pero sí señalamos la necesidad de iniciar estudios sobre el particular.

Es posible establecer una relación entre lugar de destino, actividad por realizar y cantidad de migrantes involucrados, para llegar a hablar del posible impacto social de los flujos migratorios en los lugares de destino, al menos en el Soconusco; sin embargo, por el momento esta tarea será limitada, debido al difícil acceso a las fuentes y a los informantes. Un punto de partida en este sentido es el reconocimiento de que las principales actividades económicas en la región fronteriza, y por lo tanto, potenciales generadoras de ocupación, son la agricultura, la ganadería y el comercio (ventas de artículos y prestación de servicios).

Creemos que el grueso de las migraciones centroamericanas no se dirige a residir ni a trabajar a los centros urbanos y semiurbanos de Chiapas, sino que tiene otros destinos, a reserva de una ulterior comprobación cuantitativa. Ello implica que los problemas sociales que se suscitan en las áreas urbanas chiapanecas responden a otras razones, entre las cuales tendrían que considerarse: el rápido crecimiento de ciudades como Tapachula, especialmente en los últimos decenios; la adecuación o no de las estructuras sociales a los requerimientos emergentes por los cambios sociales ocurridos en los últimos veinte años, no sólo por el crecimiento poblacional ${ }^{8}$, sino también por

${ }^{8}$ La ciudad de Tapachula ha registrado una tendencia ascendente en su ritmo de crecimiento durante los últimos años. Las cifras disponibles registran tasas promedio de crecimiento anual de $3.32,3.97$ y $3.41 \%$ para los últimos tres períodos intercensales ( $50-60,60-70$ y $70-80)$, muy por encima de las correspondientes al promedio del estado (de $2.89,2.69$ y $2.75 \%$ para los mismos períodos), lapso durante el cual su población pasó de 30,054 habitantes (1950) a 65,971 (1980). 
la mayor actividad comercial y de servicios, tanto de carácter regional como internacional; el establecimiento y mayor uso de vías de comunicación (comercio, transporte, intercambio); el incremento en losíndices de violencia, prostitución ysubempleo. En este último rubro se señala el caso de mujeres y niños que practican el comercio ambulante, sobre todo en Tapachula, pero cuyo número es relativamente limitado; también se cita el caso de mujeres que se ocupan como trabajadoras domésticas y en la prostitución, pero dado que no hay registros oficiales, no se puede hablar de un número ni tampoco de la nacionalidad de las prostitutas; se habla también de los niños que se dedican a lustrar zapatos en las plazas públicas, pero su número es también menor e igualmente impreciso

\section{LAS MIGRACIONES AL CAMPO CHIAPANECO}

Ahora veremos los efectos de la migración sobre las demandas en el campo, especialmente en lo que se refiere a la dotación de tierras para la producción, así como algunos aspectos relativos a las particularidades que presenta la migración a las zonas fronterizas. Se especificaron las condiciones en que los migrantes guatemaltecos, en especial los trabajadores del campo, llegan a Chiapas a trabajar y con qué intención.

En primer término, tenemos que considerar su condición migratoria, puesto que no podemos olvidar que, a pesar de las semejanzas étnicas, sociales y culturales, se trata de extranjeros y eventualmente indocumentados. Así, es difícil pensar que tengan la intención de adquirir legalmente una parcela en territorio mexicano, por un importante factor en su contra: descartamos la idea de que puedan comprarla, debido a que no cuentan con los recursos económicos suficientes ${ }^{9}$, o en caso de que los tuvieran, seguramente preferirían hacerlo en su país. Tampoco existe ninguna validez en afirmar que los centroamericanos

${ }^{9}$ Decimos que no cuentan con los recursos necesarios para adquirir tierras con base en los siguientes hechos detectados en los resultados de nuestra investigación de campo: 1. En general, se trata de campesinos muy pobres que, en el mejor de los casos, poseen una pequeña extensión de tierra, eso sí, insuficiente y poco productiva; 2 . En el caso de jornaleros sin tierras, sus movimientos migratorios están determinados por la oportunidad de obtener un ingreso en dinero, ya que los datos sobre sus remuneraciones en los lugares de residencia permanente muestran rasgos de insuficiencia y, por lo tanto, incapacidad de ahorro como para pensar en la disponibilidad de un pequeño capital para adquirir tierras. 
vienen al campo mexicano con el propósito de invadir tierras. Si ésta es una práctica riesgosa para los propios campesinos mexicanos, pues fácilmente pueden ser desalojados, para los extranjeros el riesgo es aún mayor: en su condición serían sujetos de encarcelamiento y deportación con todas las consecuencias subsecuentes. Lo que no podemos dejar de reconocer es que, en efecto, los migrantes centroamericanos, y en especial los guatemaitecos, llegan a la frontera sur de México; pero veamos en qué condiciones llegan, su intención, a qué se dedican y en qué tiempo lo hacen.

Antes de avanzar, conviene apuntar que la búsqueda de fuentes documentales que le dan sustento a las concepciones alarmistas resulta poco exitosa. En general, los estudios son escasos; existe uno que otro registroy uno que otro estudio particular, pero con poca profundidad sobre las características de algún flujo migratorio y casi nada sobre su impacto social en las localidades chiapanecas a donde llegan, excepción obvia de los refugiados guatemaltecos arribados desde 1982, de los cuales ya hemos hablado.

Existen cuatro puestos importantes de control migratorio a lo largo de la frontera mexicana con Guatemala en el estado de Chiapas, desde los cuales se regula el tránsito transnacional vía carreteras yaeropuerto, así como también supuestamente el paso por las múltiples veredas. Sin embargo, estos puestos fronterizos, en la práctica, no pueden controlar el paso a pie, a causa del terreno montañoso de la región, por lo que una cobertura total resultaría muy costosa.

Para el caso de los trabajadores temporales, dada la tradición del flujo y los usos que se han tornado costumbre, las autoridades han tratado de llegar a algunos acuerdos con los residentes de esas regiones, con el objeto de regularizar la situación y mejorar los registros y controles; por ejemplo, con los finqueros y ejidatarios para tratar de documentar a sus empleados. Pero, aún así, las autoridades migratorias se quejan de que han encontrado muchos trabajadores indocumentados laborando irregularmente, y en esas circunstancias se pueden aducir argumentos legales para proceder a su deportación; ello propiciaba que, en ocasiones, los trabajadores se tuvieran que ir sin haber recibido la paga correspondiente a las labores realizadas dandolugar a conflictos de mayor envergadura. 
Por otro lado, las autoridades trataron de realizar un programa de construcción de albergues para que esta población tuviera dónde guarecerse en los períodos de espera a ser contratados, pues tenían que permanecer días y noches a la interperie. Para financiarlo se requirió a cada uno de los dueños de las plantaciones que pagaran un mil pesos por cada trabajador, con el propósito de contribuir a los gastos de legalización y la construcción de los albergues ${ }^{10}$.

Veamos ahora cuáles son las características de los trabajadores estacionales que nos permiten afirmar que se trata de trabajadores del campo y para los cuales el trabajo agrícola temporal constituye una parte de sus mecanismos habituales de reproducción. Una advertencia necesaria es que la información sobre el particular adolece de las mismas deficiencias que para el resto de los movimientos migratorios. Esta carencia ha permitido que, en determinadas circunstancias, se maneje una amplia gama de cifras sin ningún referente concreto y confiable; sin embargo, se han hecho algunos esfuerzos por estimar los límites posibles del flujo, pero no existe realmente ninguna base de sustentación para las diversas aseveraciones ${ }^{11}$.

En la región del Soconusco, que es el área en la que se realizó el trabajo de investigación, el cultivo más importante es el café. Aunque

${ }^{10}$ En la época en que presenciamos la puesta en práctica de dichas medidas, se presentaron ciertas dificultades entre autoridades y algunos propietarios privados y ejidatarios; podemos afirmar que, de acuerdo a los resultados inmediatos, no se alcanzaron en su totalidad las expectativas. No obstante, sabemos que, a la fecha, ya se han realizado algunas de dichas construcciones, pero no contamos con información acerca de la continuidad de la política y el progreso en otorgar y velar porque se presten mejores condiciones de vida y de trabajo a los migrantes. En esa misma línea, desde fines de 1986 se inició un programa oficial de crédito a los productores de café, para mejorar el alojamiento de los trabajadores estacionales en el interior de las unidades productivas.

${ }^{11}$ Funcionarios, dirigentes campesinos, medios de comunicación, líderes políticos, etc. han expresado diversas magnitudes que oscilan en un margen sumamente amplio, que por su misma extensión (desde 30,000 hasta 150,000 ) no constituyen un referente útil ni confiable. La declaración más reciente que conocemos es la del delegado regional de la Dirección General de Servicios Migratorios, quien afirmó el año pasado que llegarían 40 mil trabajadores guatemaltecos a la cosecha del café 1987/ 88. En el informe final del trabajo de investigación de A. Mosquera (1986), se incluye un cálculo de los posibles montos de migrantes anuales, a partir de una estimación de los requerimientos de mano de obra, con base en algunos supuestos acerca de la productividad de las extensiones cultivadas de café en la región del Soconusco. 
sc desarrollan otras actividades productivas en el agro (ganadería, uzicar, soya, etc.), la mayor fuente de ocupación la constituyen las plantaciones del primer cultivo. Las características de su proceso productivo requieren un elevado volumen de fuerza de trabajo, cspecialmente en el período de cosecha, y de allí que la migración estacional de trabajadores nacionales y extranjeros posea una larga tradición. Durante mucho tiempo, los campesinos de los Altos de Chiapas concurrieron a estas labores a la par de sus homólogos guatemaltecos. Sin embargo, las observaciones recientes -no mediciones ni registros rigurosos- afirman que el primero de los flujos ha tendido a desaparecer. No podemos, con la información disponible, cstablecer las causas precisas de este cambio en el patrón migratorio; sin embargo, creemos necesario realizar un estudio específico acerca del nuevo comportamiento de los antiguos grupos de migrantes chiapanecos $^{12}$.

Según los resultados de la encuesta ya mencionada, en la que se entrevistaron 591 trabajadores temporales que se emplearon en unidades productoras de café (privadas y ejidales) en la cosecha 1986/7, la mayoría (un $97.8 \%$ ) había trabajado en labores del campo en sus lugares de origen en el momento previo a su venida, pero además aportó información que confirma que siempre han trabajado en el campo, incluyendo también sus diferentes estadías previas en México.

12 La explicación fácil y difundida de este fenómeno es que el segundo grupo de migrantes ha desplazado al primero, ya que se dice que está dispuesto a aceptar inferiores condiciones de trabajo y de vida en las plantaciones de café. Pensamos que ello no es necesariamente cierto y que habría que expiorar la situación actual de los antiguos migrantes y sus descendientes para establecer si el trabajo migratorio aún cumpliría la misma función complementaria en sus procesos de producción. En este sentido, habría que ubicar el análisis en el marco de cambios en la dinámica económica y social en Chiapas en las úitimas décadas. Así, habría que considerar el impacto regional y subregional, entre otros elementos, de los proyectos de desarrollo ganadero extensivo, de colonización de la zona selva (Lacanđona), de construcción de grandes obras de infraestructura (principalmente hidroeléctrica), de desarrollo petrolero a partir de fines de la década pasada en el norte de Chiapas y la vecina Tabasco, de mejoramiento de los sistemas y la infraestructura de comunicaciones intra e interregionales y de crecimiento urbano ligado a otras actividades (por ejemplo, los casos de Tuxtia Gutiérrez, Tapachula y San Cristobal de las Casas, por sus funciones comerciales, burócratas y de servicios). 
Su origen rural y su condición de trabajadores del campo se comprueba en parte por la información que proporcionaron respecto a su ocupación dentro de las unidades productivas en el momento de la entrevista (cuadro 2), pero también por el hecho de que la gran mayoría $(97.8 \%)$ de los entrevistados que indicaron haber tenido trabajo en el periodo previo a su venida, lo hicieron en actividades de carácter agrícola.

El proceso de trabajo de la producción de café en el momento de la cosecha exige precisamente una mayor concentración de trabajadores dedicados al corte del grano. De allí que la mayoría de entrevistados mencionaron esta actividad como la principal ocupación en el momento de la entrevista (ver cuadro 2).

Cuadro 2. Ocupación de los trabajadores migratorios al momento de la entrevista en la unidad productiva (en porcentajes).

1. Cortador de café

2. Trabajador en el beneficio

3. Preparador de comida

4. Caporal

5. Labores diversas en el campo

6. Labores diversas en el casco de la finca
$81.5 \%$

$7.2 \%$

$2.2 \%$

$1.2 \%$

$5.3 \%$

$2.6 \%$

T O T A L

$100.0 \%$

(586)

Fuente: Resultados de las entrevistas del proyecto "Impactos regionales de las migraciones internacionales a la frontera sur de México. El caso de los trabajadores estacionales". Centro de Estudios Demográficos y de Desarrollo Urbano. El Colegio de México.

Aunque para algunas personas el corte del café podría parecer una tarea fácil y que no requiere ninguna especialización, en la 
práctica encontramos que ello no es así. En primer lugar, las condiciones topográficas y climatológicas en que se trabaja son difíciles y agotadoras, de manera que hacen pesado no sólo el trabajo de corte, sino también la transportación de los voluminosos bultos que constituyen el producto de la tarea diaria; en general, el área de plantación se ubica en lugares inaccesibles a vehículos que, en el caso de otros cultivos, facilitan la movilización de los bultos. Por otra parte, el corte requiere un conocimiento del grado de madurez, del manejo de la planta para evitar su maltrato y, en fin, una familiaridad y destreza que sólo brinda la experiencia.

Por otro lado, en nuestras observaciones se pudo comprobar que los trabajadores migratorios que acuden a las plantaciones a realizar dichas labores, poseen una larga tradición de participación en el trabajo estacional requerido en las cosechas de café. El patrón de distribución de frecuencias del cruce de las variables edad y tiempo transcurrido desde que vino por primera vez a trabajar a México, es coherente con algunos hechos esperables: a) las personas de mayor edad muestran los mayores períodos transcurridos desde que llegaron por primera vez, e incluso encontramos personas mayores (de más de 65 años) con una larga historia de migraciones que sorprende por lo pesado y arduo de las tareas; b) las personas jóvenes acusan menores frecuencias, pero desde ya se detecta una tendencia continua e ininterrumpida en su presencia a laborar en la cosecha del café (ver cuadro 3), pero también muestra que las migraciones laborales, en general, se inician desde edades muy tempranas.

Sin embargo, podemos avanzar en el análisis para demostrar el carácter cíclico de la migración si hacemos una diferenciación por grupos de edades. Para ello, se construyó un indicador ${ }^{13}$ que permitiera estimar el número de migraciones que en promedio han realizado los entrevistados a lo largo de su historia de desplazamientos. El resultado global arroja un indicador promedio de 1.05 veces por año y los parciales muestran una tendencia a incrementarse el número de los grupos más jóvenes, lo cual se podría interpretar como una tendencia

13 Se utilizó un indicador del promedio de veces que los entrevistados de un grupo de edad han venido a trabajar a México y con base en él, se construyó un Indice Medio de Ciclicidad. 


\section{CRITICA A LOS MITOS ACERCA DE LAS MIGRACIONES}

a consolidar una mayor continuidad y frecuencia de su participación en las diferentes labores del café ${ }^{14}$.

CUADRO 3. Proporción de trabajadores migratorios por intervalos de edad y de tiempo transcurrido desde que vino por primera vez a trabajar a México, según intervalos que se indican para cada caso (en porcentajes).

\begin{tabular}{|c|c|c|c|c|c|c|}
\hline \multirow{2}{*}{$\begin{array}{l}\text { Tiempo } \\
\text { transcurrido } \\
\text { desde que } \\
\text { vino por } \\
\text { primera vez } \\
\text { a trabajar a } \\
\text { México ( en } \\
\text { años ) }\end{array}$} & \multicolumn{6}{|c|}{ Intervalos de edad (en años) } \\
\hline & $12-19$ & $20-29$ & 30-39 & $40-59$ & $\begin{array}{l}50 \text { y } \\
\text { más }\end{array}$ & $\begin{array}{c}\text { Totales } \\
\text { renglo- } \\
\text { nes }\end{array}$ \\
\hline 0 (1ra.vez) & 1.1 & 1.4 & 0.5 & 0.2 & 0.2 & 3.5 \\
\hline $1-3$ & 9.7 & 7.6 & 1.7 & 1.1 & 0.9 & 21.0 \\
\hline $4-6$ & 6.4 & 7.0 & 1.7 & 0.7 & 0.4 & 16.2 \\
\hline $7-11$ & 3.3 & 8.2 & 2.8 & 1.4 & 0.5 & 16.2 \\
\hline $12-17$ & 1.2 & 7.1 & 6.1 & 1.1 & 0.5 & 16.0 \\
\hline $18-28$ & - & 0.5 & 8.5 & 4.9 & 1.9 & 15.8 \\
\hline 29 y más & - & - & 0.7 & 5.7 & 4.9 & 11.3 \\
\hline TOTALES & 21.7 & 31.8 & 22.0 & 15.1 & 9.3 & 100.0 \\
\hline
\end{tabular}

Fuente: Resultados de las entrevistas del proyecto "Impactos regionales de las migraciones internacionales a la frontera sur de México. El caso de los trabajadores estacionales". Centro de Estudios Demográficos y de Desarrollo Urbano. El Colegio de México.

14 Aunque ya dijimos que los trabajadores temporales del café se ocupan principalmente en el corte del grano, lo cual ocurre en el período de cosecha -que puede variar entre dos y tres meses en cada plantación en promedio-, también se utiliza una menor proporción de fuerza de trabajo temporal a mediados del año cuando se requiere realizar labores de limpia y cuidado de la plantación previas a la cosecha. 
Finalmente, para concluir con nuestra argumentación, trataremos de demostrar el significado de la migración para estos trabajadores. Se mencionó antes que en nuestra indagación se encontró que la gran mayoría (prácticamente la totalidad) de los entrevistados eran trabajadores agrícolas en sus lugares de residencia. Lo importante del caso reside en señalar que, de ellos, las mayores proporciones, el $18.2 \%$ y el $69.6 \%$ respectivamente, se declararon como trabajadores familiares (y en este último porcentaje se incluye a propietarios en un $66.0 \%$, comuneros $1 \%$, cooperativas $1 \%$ y arrendatarios $1.6 \%$, ver cuadro 4). Interpretamos estos resultados en el sentido de que ambos grupos tienen, en diversas formas, acceso a la tierra productiva agrícola, aunque en un segundo momento comprobamos que las extensiones son insuficientes para las necesidades individuales y familiares según sea el caso. El 12.2\% restante se declaró comojornalero agrícola, lo cual significa que los pertenecientes a este grupo no tienen acceso directo a la tierra productiva; sin embargo, el examen de sus condiciones de trabajo asalariado en sus lugares de origen muestra que sus ingresos son insuficientes y, en muchos casos, muy por debajo del mínimo legal establecido. En principio, con la información disponible, podemos afirmar que el trabajo migratorio constituye básicamente para los tres grupos señalados, una forma de obtención de ingresos en dinero que, para el caso de los trabajadores familiares y las categorías de algún tipo de propiedad de la tierra, permiten allegarse recursos monetarios cada vez más indispensables en una economía crecientemente mercantilizada; desde el punto de vista de los jornaleros, la hipótesis sugerida es que el trabajo temporal puede resolver no solamente la necesidad de ingresos por la insuficiencia de la paga en los lugares de origen, sino también las limitaciones de fuentes de ocupación en el campo guatemalteco durante ciertos períodos del año ${ }^{15}$.

${ }^{15}$ Uno de los principales síntomas de la crisis económica que ha experimentado el vecinopaís durante la presente década es la escasez de fuentes de empleo. La CEPAL señala que, si bien la tendencia ascendente de las tasas de desocupación abierta registrada desde 1982 pudo iniciar un principio de reversión, al disminuir ligeramente este indicador de $14.25 \%$ en 1986 a $12.6 \%$ en 1987 (con base únicamente en los registros del sistema de seguridad social), el subempleo se mantuvo en casi $32 \%$ (incrementando su magnitud en términos absolutos); pero además, dice que "En el ámbito rural, los problemas del empleo tienden a agravarse debido al debilitamiento que experimentaron algunos productos agroexportadores de uso intensivo de mano de obra..." (Cfr. Cepal, 1988: 10). 
CUADRO 4. Posición en el trabajo principal de los trabajadores agrícolas migratorios (en porcentajes).

\begin{tabular}{lc}
\hline Posición en el trabajo principal & $\%$ \\
\hline 1. Propietario de tierra & 66.0 \\
2. Comunero & 1.0 \\
3. Cooperativista & 1.0 \\
4. Arrendatario & 1.6 \\
5. Peón o Jornalero & 12.2 \\
6. Trabajador familiar & 18.2 \\
\hline T O T A L & 100.0 \\
& $(494)$ \\
\hline
\end{tabular}

Fuente: Resultados de las entrevistas del proyecto "Impactos regionales de las migraciones internacionales a la frontera sur de México. El caso de los trabajadores estacionales" Centro de Estudios Demográficos y de Desarrollo Urbano. El Colegio de México.

\section{CONCLUSIONES}

En el presente artículo se ha querido realizar un primer ejercicio de contraste entre algunas de las creencias que se han difundido en diversos sectores de la sociedad mexicana -que incluso han trascendido o han sido exacerbadas desde fuera de sus fronteras- acerca de ciertos aspectos de las migraciones centroamericanas hacia su frontera sur. No hemos abordado a propósito todos los elementos citados al inicio, principalmente dentro de los supuestos que señalamos como constituyentes del trasfondo de los mitos más extendidos según nuestra percepción. Algunos de ellos tienen que ver más con situaciones de carácter ideológico, como son las acusaciones que se les hace a los migrantes acerca de su actividad y vinculaciones de orden político; 
creemos que en el caso de los refugiados dichas acusaciones han sido ampliamente desvanecidas.

Pensamos más bien que, con los elementos de que disponemos principalmente a partir de la investigación, hemos sustentado nuestra opinión de que debido, entre otras razones, a la carencia de conocimiento riguroso del fenómeno, resulta fácil propagar ideas erróneas, a veces convenientes para los intereses de ciertos sectores, sobre todo en medios y momentos propicios como a los que ha dado lugar la crisis en sus diversas expresiones económico, político y sociales ${ }^{16}$.

En síntesis, creemos haber aportado elementos para demostrar que, si bien por una parte se ha hecho más complejo el fenómeno migratorio en la frontera sur, no existen evidencias suficientes para señalar su magnitud con algún grado de certeza. También consideramos que buena parte de la inquietud que se ha generado en torno al tema durante la presente década, se debe a los flujos generados por la situación de conflictoen la vecina región centroamericana, cuya mayor expresiónla encontramos en el grupo de refugiados guatemaltecos que llegaron en una cantidad no determinada. Sin embargo, aquéllos que se localizaron en campamentos fueron objeto de atención de las comunidades nacional e internacional, buscando la manera de que su permanencia en el país no constituyera una dificultad adicional significativa.

Por otra parte, hemos tratado de demostrar, con un análisis de algunos de los resultados de nuestra investigación, que en la migración más tradicional -la de trabajadores estacionales a las plantaciones de café del Soconusco- se encuentran rasgos que comprueban su carácter cíclico, la importancia que esa actividad posee para los mecanismos de reproducción de los trabajadores y sus familias, así como el hecho de que la principal ocupación que desempeñan durante el tiempo que permanecen en las unidades productivas del área confirman un cierto grado de especialización.

16 Un ejemplo evidente de ello lo constituye la tan difundida idea de que hay que ponerle un alto a la inmigración centroamericana, puesto que agrava las demandas en torno a la generación de fuentes de empleo que, en el momento actual, no son suficientes para los nacionales. 
Por último, queremos enfatizar que la carencia de conocimientos requiere estudios más profundos pero también diversos, es decir, que se enfoquen al análisis de los otros flujos migratorios que por el momento no hemos abordado en este trabajo. Sabemos que existe una corriente con alguna tradición que no sólo se dirige al territorio mexicano en busca de mejores condiciones de vida y de trabajo, sino que probablemente en sumayoría se encaminan hacia los Estados Unidos por las expectativas que ofrece la poderosa economía norteamericana. Es de esperar que mientras continúen prevaleciendo las condiciones críticas -no sólo en lo económico, sino principalmente en lo político y en el respeto a los derechos humanos- que han vivido los países centroamericanos, especialmente durante los últimos años, este movimiento continuará ocurriendo; en todo caso, la forma adecuada de enfrentar este fenómeno no radica en el incremento de las medidas de control, sino más bien en la oportuna contribución para solucionar los problemas de origen. 


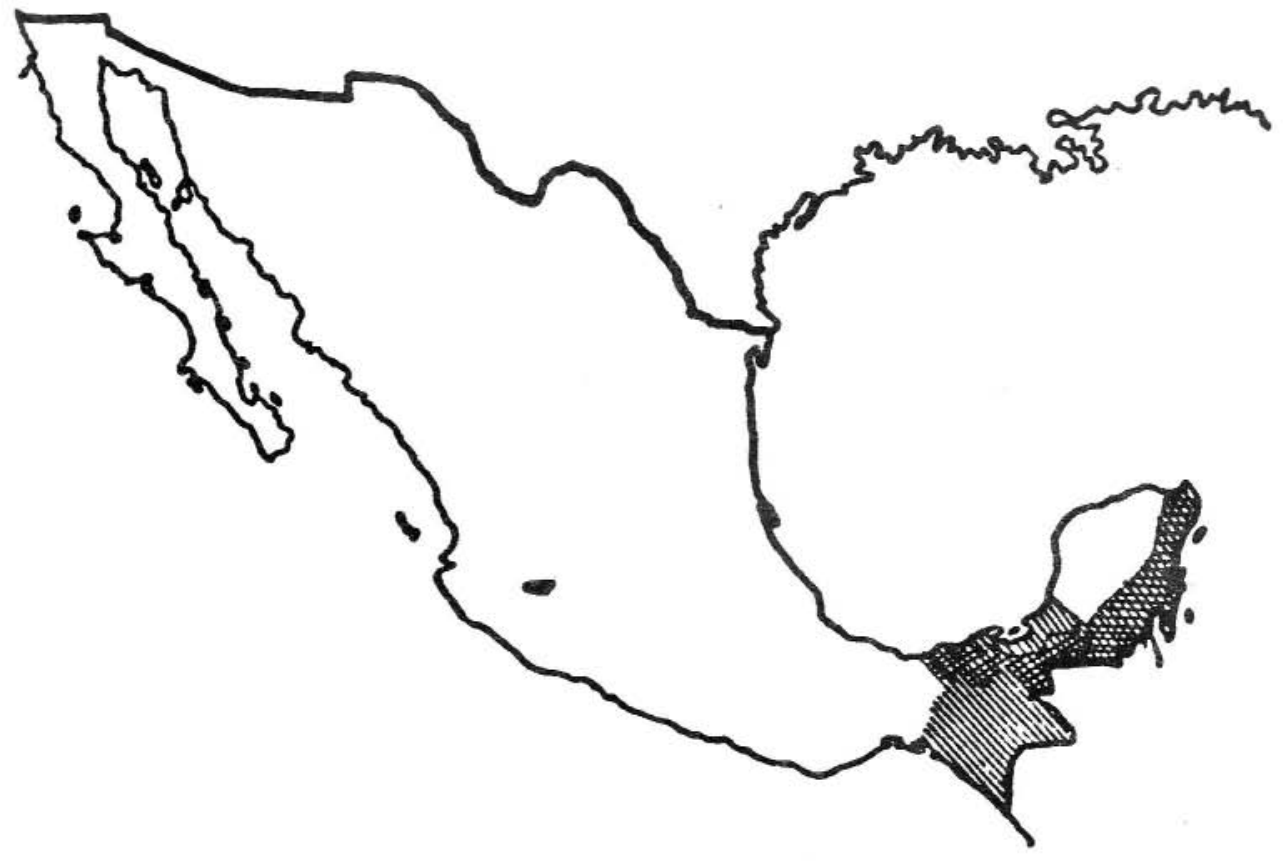

Fuente: Elaboración propia.

FIGURA 1. República mexicana y estados de la frontera sur. 


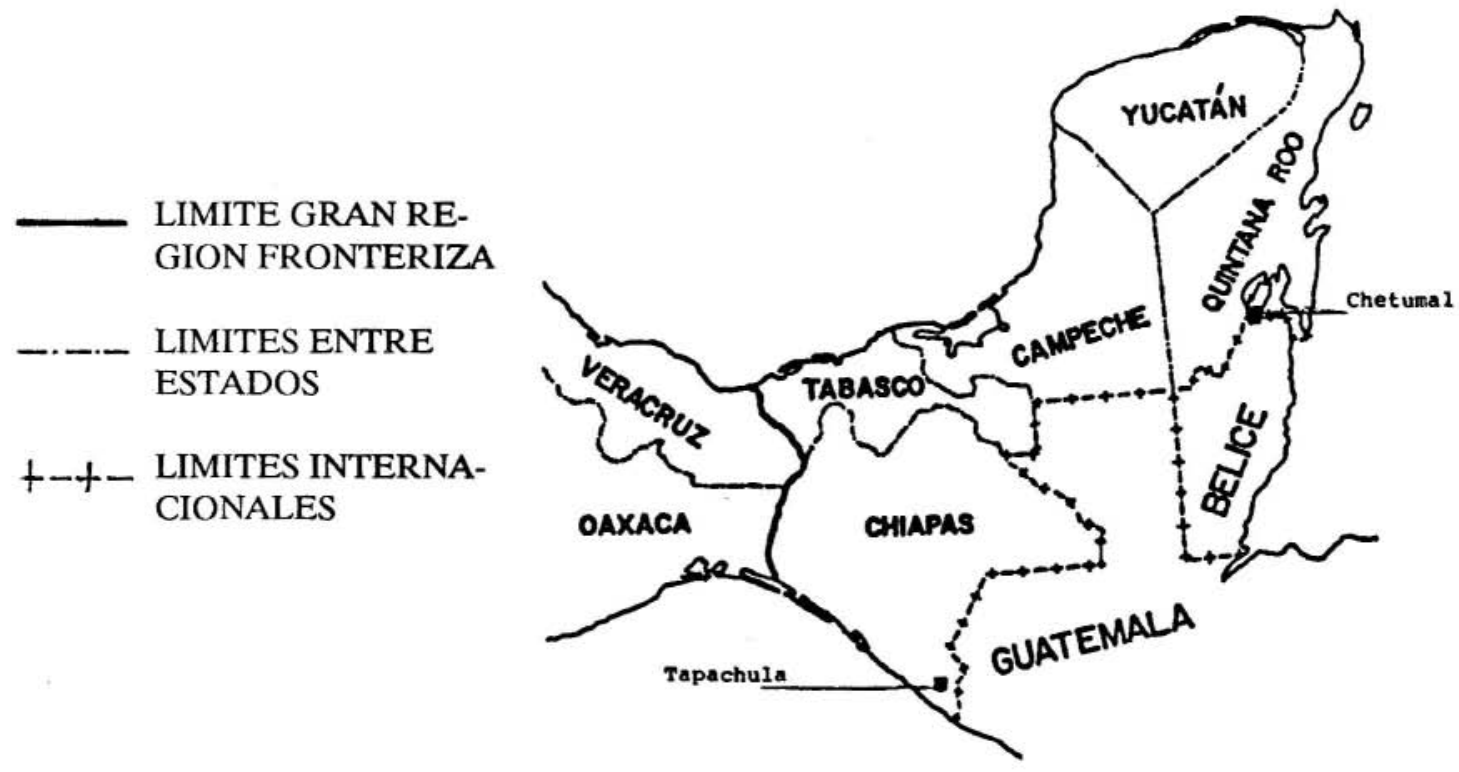

Fuente: Fábregas, A et al.: La formación histórica de la frontera sur, Cuadernos de la Casa Chata 124, CIESAS del Sureste, México, 1985, p. 99.

FIGURA 2. La región fronteriza del sur de México 

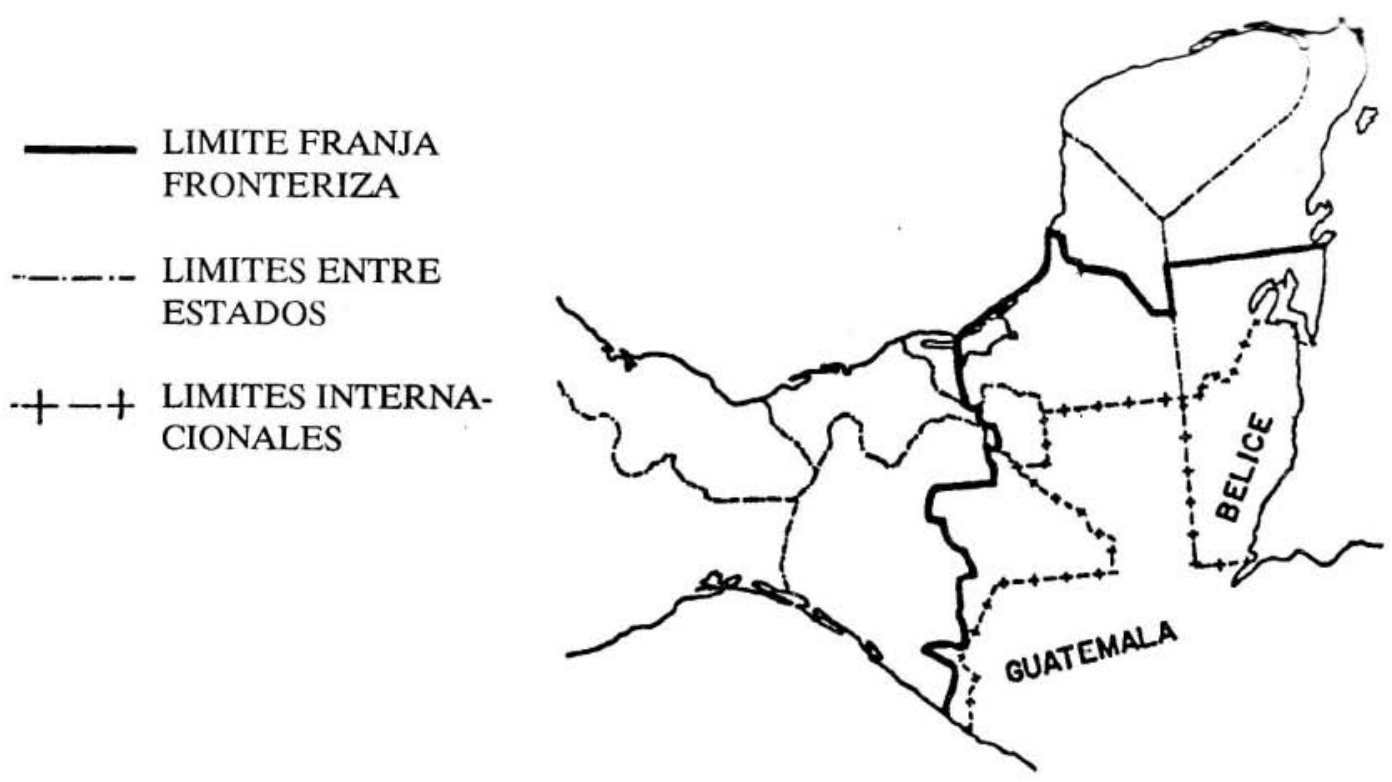

Fuente: Fábregas, A. et al.: La formación histórica de la frontera sur, Cuadernos de la Casa Chata 124, CIESAS del Sureste, México, 1985, p. 95.

FIGURA 3. La franja fronteriza sur de México 


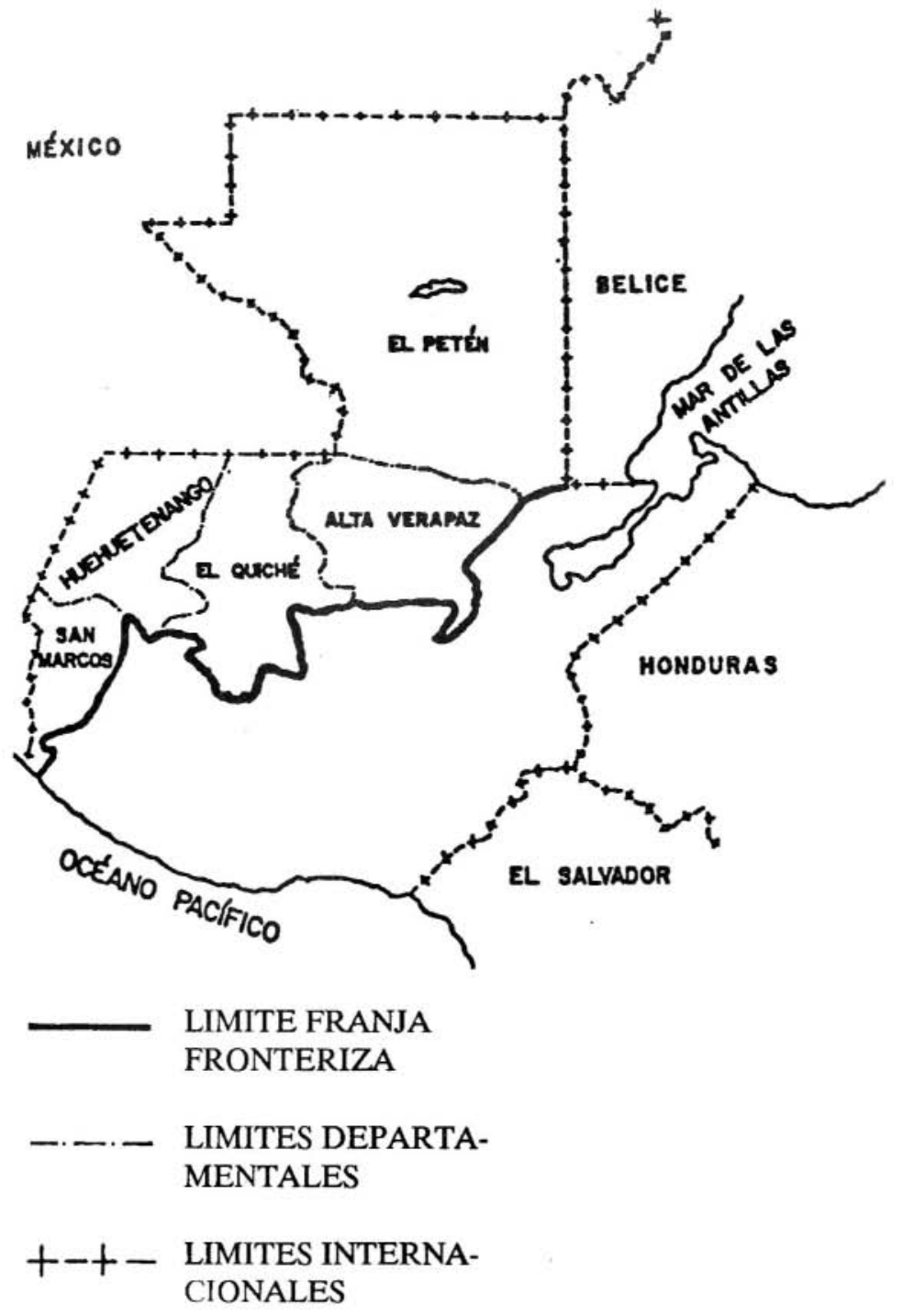

Fuente: Fábregas et al:: La formación histórica de la frontera sur, Cuadernos de la Casa Chata 124, CIESAS del Sureste, México, 1985, p. 97 . Tomado a su vez de IGN. Mapa esquemático para turismo y carreteras. Guatemala, 1978. Com. Int. de Límites y Aguas entre México y Guatemala. Mapa base de las cuencas de los ríos internacionales. México, 1980.

FIGURA 4. La franja fronteriza Guatemalteca con México 


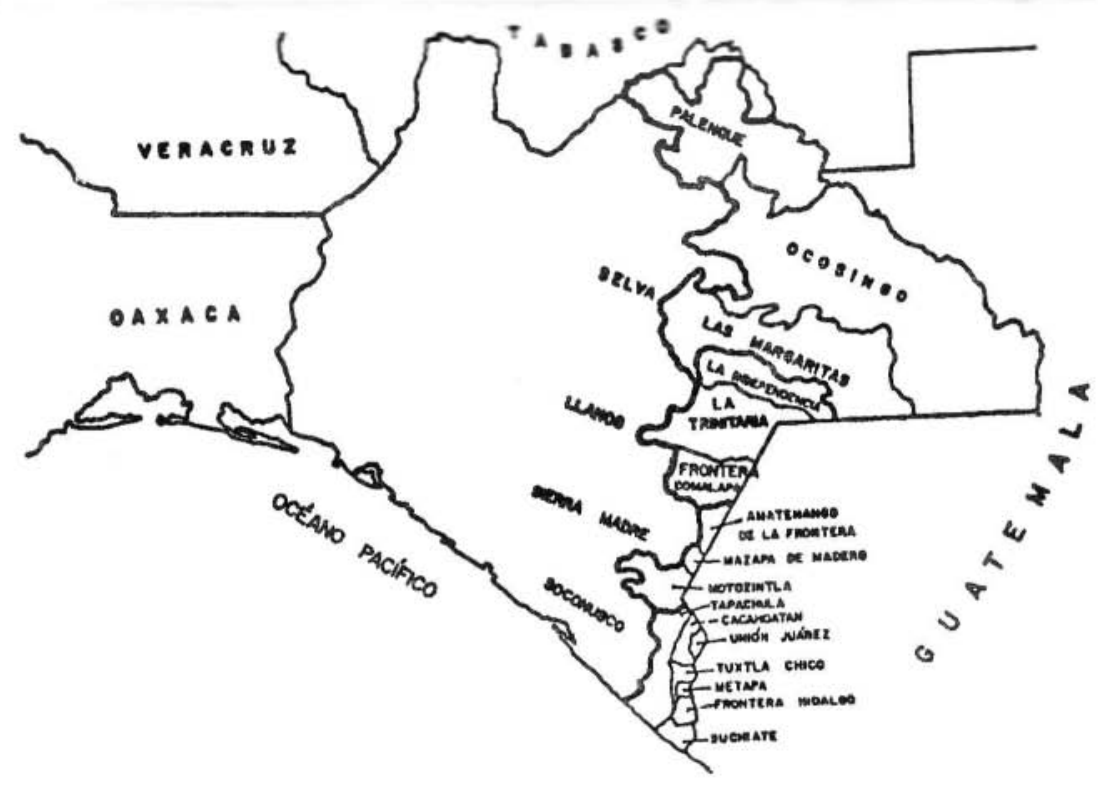

Fuente: Fábregas et al.: La formación histórica de la frontera sur, Cuadernos de la Casa Chata 124, CIESAS del Sureste, México, 1985, p. 101.

FIGURA 5. Las regiones fronterizas de Chiapas. 


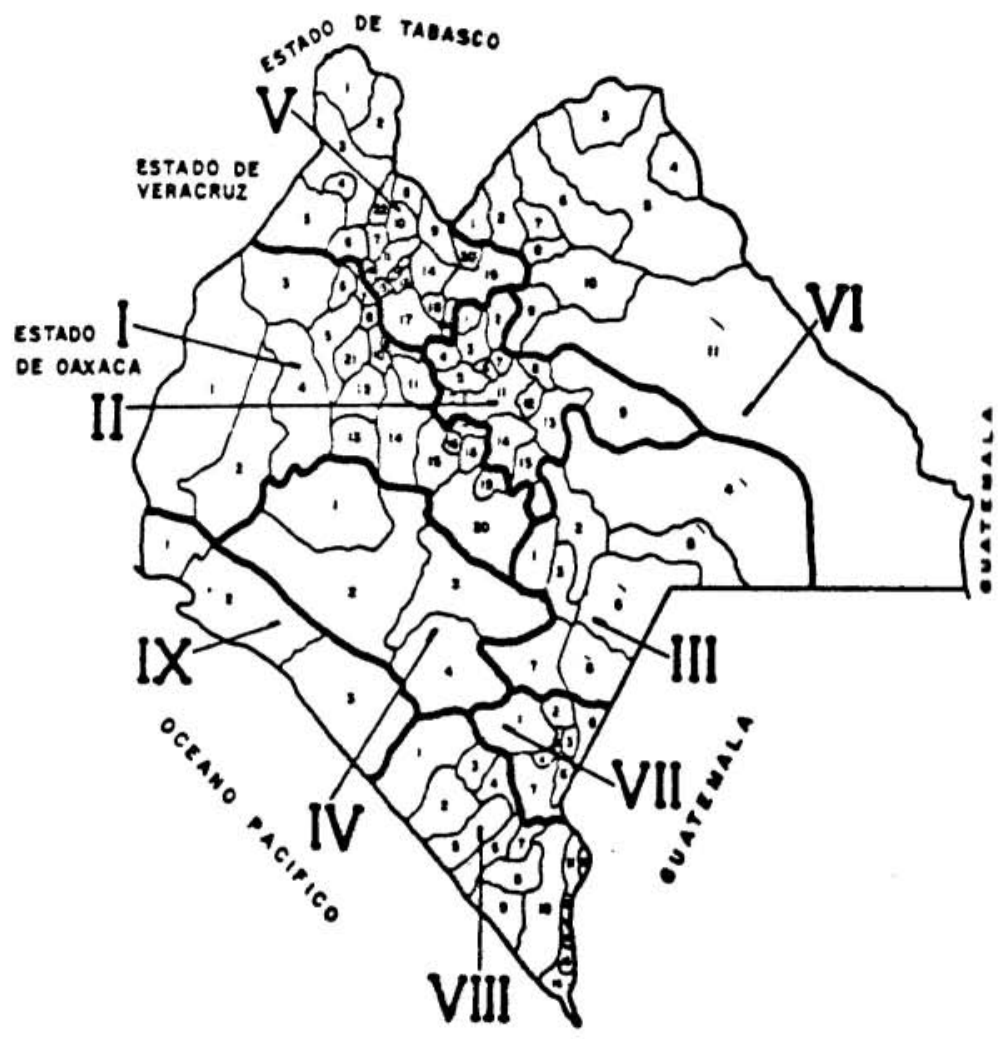

Fuente: Consejo Nacional de Población: Estudio sociodemográfico del estado de Chiapas, CONAPO Consejo de Población del Estado de Chiapas, México, 1985, p. 30. Tomado a su vez de Gobierno del Estado de Chiapas. Chiapas plan y programas de gobierno, 1982-1988, México, 1982.

FIGURA 6. División municipal y regional del estado de Chiapas, 1980 


\section{B I B L I O G R A F I A}

AGUAYO, S. 1985. El éxodo centroamericano. CONAFE/SEP. México.

AGUAYO, S. y L. O'Dougherty. 1986. "Los refugiados guatemaltecos". En: Foro Internacional. No. 106. Centro de Estudios Internacionales, Vol. XXVII, No.2. El Colegio de México. México. Oct.-dic.

CASILLAS, R. y M.A. Castillo. 1987. "Mitos y habilidades sobre las migraciones centroamericanas a Chiapas". Ponencia presentada en el Foro El redescubrimiento de la frontera sur de México. Universidad Autónoma Metropolitana/Departamento de Etnología y Antropología Social (INAH) México, D.F. Noviembre 1987. (Mimeo).

CEPAL. 1988. Notas para el estudio económico de América Latina y el Caribe, 1987. CEPAL. Doc. LC/MEX/L.83, 19 de julio.

MOSQUERA, A. 1986. Los trabajadores guatemaltecos migrantes a Chiapas, México. Informe final del trabajo de investigación. Universidad Autónoma de Chiapas/Asociación Mexicana de Población. México. (Mimeo). 